But this is comprised in Mr. Salmon's theorem in his paper referred to. It can be also shown that for the twisted cubic of the type (2) of constant curvature, the same conditions (4) are necessary and sufficient. This was carried out by Mr. S. Narumi, one of our students. Hence the theorem: The twisted cubics of the type (2) of constant torsion are of constant curvature, and conversely those of constant curvature are of constant torsion; they are all imaginary and satisfy the conditions (4). A similar problem on the twisted cubics of the most general type (1), that is rather more interesting, as Prof. T. Kubota, one of our colleagues, says, remains unsolved.

February, 1919.

\title{
SOME GENERALIZATIONS OF THE SATELLITE THEORY.
}

BY PROFESSOR R. M. WINGER.

\$1. The Rational Cubic.

In a forthcoming paper* in the American Journal of Mathematics the author considers the satellite line of the cubic and some related curves. The object of the present note is to point out how the principal results there obtained can be generalized.

For the rational cubic $R^{3}$,

$$
x_{1}=3 t^{2}, \quad x_{2}=3 t, \quad x_{3}=t^{3}+1,
$$

the relation connecting a point $\tau$ and its tangential $t$ is

$$
t \tau^{2}+1=0 \text {. }
$$

And the condition that $3 n$ points lie on a curve $C_{n}$ is

$$
s_{3 n}=(-1)^{n},
$$

where $s$ refers to the product of the $t$ 's $\dagger$. From these equations we have at once the following theorems:

1. The tangentials of the points in which a $C_{n}^{\prime}$ cuts $R^{3}$ lie on a second $C_{n}$.

* "On the satellite line of the cubic," read before the San Francisco section of the American Mathematical Society, April 6, 1918. 1918.

Winger, "Involutions on the rational cubic," this Bulletin, Oct., 
2. The contacts of tangents from the points in which a $C_{n}$ meets $R^{3}$ lie on a $C_{2 n}{ }^{\prime}$ when $n$ is even-and only then.

Theorem 1 repeatedly applied yields a chain of curves of the same degree. We should naturally designate $C_{n}{ }^{\prime}$ and $C_{n}$ as primary and satellite respectively, but this terminology is hardly appropriate when $n>2$. For then each curve is one of a pencil on the same points. We shall therefore speak of the points in question as a primary set $S_{3 n}{ }^{\prime}$ and a satellite set $S_{3 n}$.

Any primary set has a unique satellite set* but a satellite set has a multitude of primaries. Let a conic $C_{2}$ cut the cubic in six points $t_{1}, t_{2}, \cdots, t_{6}$. Then $s_{6}=1$. The tangentials of these points may be arranged in two sets $\tau_{1}, \tau_{2}, \cdots, \tau_{6}$ and $-\tau_{1},-\tau_{2}, \cdots,-\tau_{6}$ such that the product of each set is unity. Call these points $A$ and $B$ and denote their products by $A_{6}$ and $B_{6}$ respectively. Selecting $6 t$ 's from points $A$ and $B$ in such a way that each subscript occurs but once, the set will lie on a conic provided it contains an even number of points $B$. There are thus 32 primary conics in all. Or, the twelve contacts of tangents from the points of intersection of a conic and a rational cubic lie by sixes on thirty-two conics.

In general, $n$ even, a satellite set $S_{3 n}$ will have as many primary sets as there are combinations of $3 n$ things $0,2,4, \cdots$, $3 n$ at a time. The total number is therefore the sum of alternate binomial coefficients, beginning with the first, of degree $3 n$, † i.e., $2^{3 n-1}$.

When $n$ is odd, group the points $\tau$ so that $A_{3 n}=1, B_{3 n}=$ - 1. Then points $B$ but not $A$ are on a $C_{n}$. We shall get a primary set as before, provided we use an odd number of points $B$. The total number of such sets therefore is the sum of alternate binomial coefficients of degree $3 n$ beginning with the second, again $2^{3 n-1}$.

Hence in either case, the $6 n$ contacts of tangents from the intersections of $C_{n}$ and $R^{3}$ can be arranged in primary sets $S_{3 n}^{\prime}$ in $2^{3 n-1}$ ways.

\section{\$2. The General Cubic.}

Theorem 1 of the previous section can be extended at once to the general cubic by the theory of residuation. Theorem 2 is replaced by

\footnotetext{
* If theorem 1 is applied to the points of intersection of $R^{3}$ and $C_{2 n}{ }^{\prime}$ of 2 , the satellite set $S_{6 n}$ is an $S_{3 n}$ repeated.

$\dagger$ i.e., in the expansion of a binomial of degree $3 n$.
} 
3. The $12 n$ contacts of tangents from the points in which $a C_{n}$ meets the general cubic lie on $a C^{\prime}{ }_{4 n}$.

For if the elliptic argument $u$ is properly chosen, the intersections $u_{i}$ of $C_{n}$ satisfy the condition

$$
u_{1}+u_{2}+\cdots+u_{3 n}=\mu \omega+\mu^{\prime} \omega^{\prime}{ }^{*}
$$

The contacts of tangents from $u_{i}$ are

$$
-\frac{u_{i}}{2},-\frac{u_{i}+\omega}{2},-\frac{u_{i}+\omega^{\prime}}{2},-\frac{u_{i}+\omega+\omega^{\prime}}{2},
$$

the sum of which $(i=1, \cdots, 3 n)$ is $-2 \Sigma u_{i}-3 n\left(\omega+\omega^{\prime}\right)$, hence the theorem is proved.

Again a primary set has a unique satellite set. To enumerate the primary sets of a satellite set, consider first a cubic $C_{3}$ meeting the base curve in nine points $u_{1}, u_{2}, \cdots, u_{9}$. Denote the contacts of tangents from points $u_{i}$ by $A_{i}, B_{i}, C_{i}$ and $D_{i}$. A cubic $C_{3}^{\prime}$ on eight of these points $\alpha_{1}, \cdots, \alpha_{8}, \dagger$ where the subscripts are all different, will cut again in a ninth point, say $u$. The satellite set of $C_{3}{ }^{\prime}$ will be cut out by a cubic on the eight points $u_{1}, \cdots, u_{8}$. But any cubic on these eight points passes through $u_{9}$. Hence $u_{9}$ is the tangential point of $u$, or $u$ is none other than a point $\alpha_{9}$. That is to say, any cubic on eight of the points $\alpha$, so chosen that all subscripts except one are represented, will pass through one of the four points carrying the omitted subscript and will have points $u_{i}$ for a satellite set. Now the points $\alpha_{1}$ can be chosen in four ways, $\alpha_{2}$ likewise and so on to $\alpha_{8}, \alpha_{9}$ then being determined. Hence the points $A, B, C, D$ can be chosen as primary sets in $4^{8}$ ways. By precisely the same reasoning, the contacts of tangents from the $3 n$ points in which a $C_{n}$ cuts $C_{3}$ can be arranged in primary sets $S_{3 n}^{\prime}$ in $4^{3 n-1}$ ways.

\section{§3. Extension to Higher Cases.}

The satellite theory, as frequently happens, can be generalized in several directions. This is plain if it is recalled that there are three factors involved-the primary (a line), the base curve (a cubic), and the tangent lines at the points of intersection. Any one, any two or all three of these elements may be generalized. Of the more interesting cases we mention

* Pascal, Repertorium, 2d ed., II, p. 393.

$\dagger \alpha_{i}$ refers to any one of the points with subscript $i$. 
the following, the validity of which can be inferred from the fundamental theorem of residuation.*

4. The $n$ tangents at collinear points of a $C_{n}$ meet again in $n(n-2)$ points which lie on a $C_{n-2}$.

5. The mn tangents to $C_{n}$ at points of intersection of a $C_{m}$ cut $C_{n}$ again in points of $a C_{m(n-2)}$.

6. Given a base curve $C_{n}$, and a primary $C_{m}$, cutting $C_{n}$ in points $P_{i}$. Then any set of $m n C_{p}$ 's cut $C_{n}$ in points of a $C_{m n p}$. If $C_{p}{ }^{(i)}$ has $k$-point contact with $C_{n}$ at $P_{i}$ the remaining $m n(n p$ - k) points constitute a satellite set.

Theorem 6 which is itself a special case of the fundamental theorem includes all the generalizations suggested. Theorems 4 and 5 repeatedly applied yield chain theorems connecting an unending series of curves.

In particular the dual of 5 , beginning with $n=1$, establishes the chain, the first link of which was obtained by another method in the earlier paper. $\dagger$

Many isolated classic theorems appear here as special cases. From 6 can be deduced readily the theorem that if $n-1$ intersections of a line with $a C_{n}$ are points $P_{k}$ with $k$-point tangents, $k>2$, the remaining intersection is a $P_{k}$. This includes as a special case the familiar theorem concerning flexes.

We shall conclude with a consideration of curves $C_{m}$ each of which has with a base cubic a $(3 m-1)$-point contact $P_{3 m-1}$. Thus eonics with quintactic points at intersections of a line $l^{\prime}$ with a rational cubic cut again in three points of a line $l$. But from each of the latter points can be drawn five quintactic conics. $\$$ These 15 quintactic points lie by threes on 25 lines $l^{\prime}$ five of which pass through each point.

Similarly the $24 P_{8}$ 's of cubics passing simply through the intersections of $l$ and $R^{3}$ determine a configuration of 24 points and 64 lines, 3 points on a line and 8 lines on a point.

Again if $l$ is replaced by a conic $C_{2}$ there will be 30 quintactic conics from points of intersection of $C_{2}$ and $R^{3}$. By reasoning analogous to that employed in $\$ 2$ it is easily established that these 30 points lie by sixes on $5^{5}$ conics $C_{2}{ }^{\prime}$.

Generally, from a point of $R^{3}$ can be drawn $(3 m-1) C_{m}$ 's each having a contact $P_{3 m-1}$ elsewhere. The $3 i$ common points

* Pascal, Repertorium, 2 d ed., II, p. 321.

† Winger, "On the satellite line of the cubic," l.c.

‡ Winger, "Involutions on the rational cubic," l.c., p. 30 . 
of $a C_{i}$ and $R^{3}$ determine thus $3 i(3 m-1)$ such points which can be arranged in primary sets $S^{\prime}{ }_{3 i}$ of the $3 i$ points in $(3 m-1)^{3 i-1}$ ways.

Passing now to the general cubic, if a $C_{m}$ have $(3 m-1)$ point contact at $u^{\prime}$ and cuts again at $u$ the relation connecting the elliptic arguments is

$$
u^{\prime}=\frac{\mu \omega+\mu^{\prime} \omega^{\prime}-u}{3 m-1} .
$$

For given $u$ there are $(3 m-1)^{2}$ values of $u^{\prime}$ since $\mu, \mu^{\prime}=1$, $2, \cdots, 3 m-1$. Thus from the common points of a conic and a $C_{3}$ can be drawn $6 \cdot 25$ quintactic conics whose 150 quintactic points lie by sixes on $5^{10}$ primary conics.

And generally from the $3 i$ common points of $a C_{i}$ and $C_{3}$ can be drawn $3 i(3 m-1)^{2} C_{m}$ 's each having a $(3 m-1)$-point contact. These $3 i(3 m-1)^{2} P_{3 m-1}$ 's can be grouped in primary sets $S_{3 i}{ }^{\prime}$ of the $3 i$ points in $3(3 m-1)^{6 i-2}$ ways.

UNIVERSITY OF WASHINGTON.

\section{CAJORI'S HISTORY OF MATHEMATICS}

A History of Mathematics. By Florian Cajori, Ph.D., Professor of the History of Mathematics in the University of California. New York, The Macmillan Company, 1919. vii +514 pp. Price, $\$ 4.00$.

THE present edition of Cajori's well known History of Mathematics is so completely revised and so considerably enlarged that it might almost be regarded as a new book. While it contains only about 100 more pages than the earlier edition,* it has about twice as much reading matter, the pages being larger and more closely printed than those of the first edition. The general arrangement of the subjects treated remains unchanged, but three brief new sections relating largely to ancient mathematics have been added. These are headed; "The Maya," "The Chinese" and "The Japanese" respectively, and are based on special histories relating to these peoples, which have appeared since the publication in 1894 of the first edition of the present work.

* Reviewed in this Bulletin, vol. 3 (1894), pp. 190 and 248, by D. E. Smith and G. B. Halsted. 